\title{
Plutonium Immobilization Project Pictures from the Can Loading Puck Can Inspection Demonstration (Milestone 2.2.4.5/FY00/b)
}

\author{
P. Maddux
}

October 16, 2000

U.S. Department of Energy

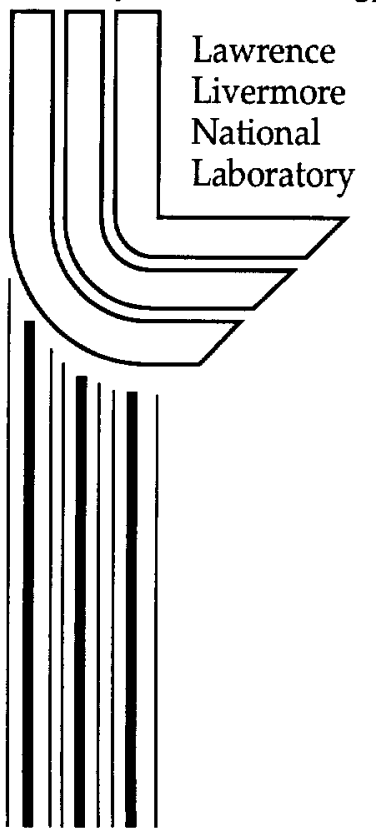




\section{DISCLAIMER}

This document was prepared as an account of work sponsored by an agency of the United States Government. Neither the United States Government nor the University of California nor any of their employees, makes any warranty, express or implied, or assumes any legal liability or responsibility for the accuracy, completeness, or usefulness of any information, apparatus, product, or process disclosed, or represents that its use would not infringe privately owned rights. Reference herein to any specific commercial product, process, or service by trade name, trademark, manufacturer, or otherwise, does not necessarily constitute or imply its endorsement, recommendation, or favoring by the United States Government or the University of California. The views and opinions of authors expressed herein do not necessarily state or reflect those of the United States Government or the University of California, and shall not be used for advertising or product endorsement purposes.

This work was performed under the auspices of the U. S. Department of Energy by the University of California, Lawrence Livermore National Laboratory under Contract No. W-7405-Eng-48.

This report has been reproduced directly from the best available copy.

Available electronically at http://www.doc.gov/bridge

Available for a processing fee to U.S. Department of Energy

And its contractors in paper from

U.S. Department of Energy

Office of Scientific and Technical Information

P.O. Box 62

Oak Ridge, TN 37831-0062

Telephone: (865) 576-8401

Facsimile: (865) 576-5728

E-mail: reports@adonis.osti.gov

Available for the sale to the public from

U.S. Department of Commerce

National Technical Information Service

5285 Port Royal Road

Springfield, VA 22161

Telephone: (800) 553-6847

Facsimile: (703) 605-6900

E-mail: orders@ntis.fedworld.gov

Online ordering: http://www.ntis.gov/ordering.htm

OR

Lawrence Livermore National Laboratory

Technical Information Department's Digital Library

http://www.llnl.gov/tid/Library.html 
FMD Program

Plutonium Immobilization

Project
Lawrence Livermore National Laboratory

October 26, 2000

PIP 00-129LTR

William J. Danker

Immobilization Project Leader

Office of Materials and Immobilization, NN-62

U.S. Department of Energy

1000 Independence Avenue, SW

Washington, DC 20585

Dear Mr. Danker:

Subject: Closeout of Milestone 2.2.4.5/FY00/b

Attached is the closeout of Milestone 2.2.4.5/FY/b, "Can Loading Robot and Can Swiping Robot Demonstration Complete."

If you or your staff have any questions regarding this evaluation, please contact me, or Paul Maddux.

Sincerely yours,

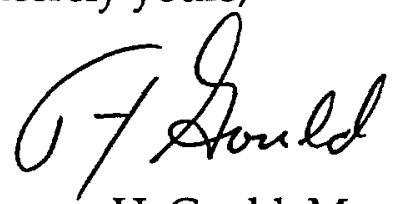

Thomas H. Gould, Manager

Plutonium Immobilization Project

Attachment 
Fissile Materials Disposition Program

Plutonium Immobilization Project Pictures from the Can Loading Puck Can Inspection Demonstration (Milestone 2.2.4.5/FY00/b)

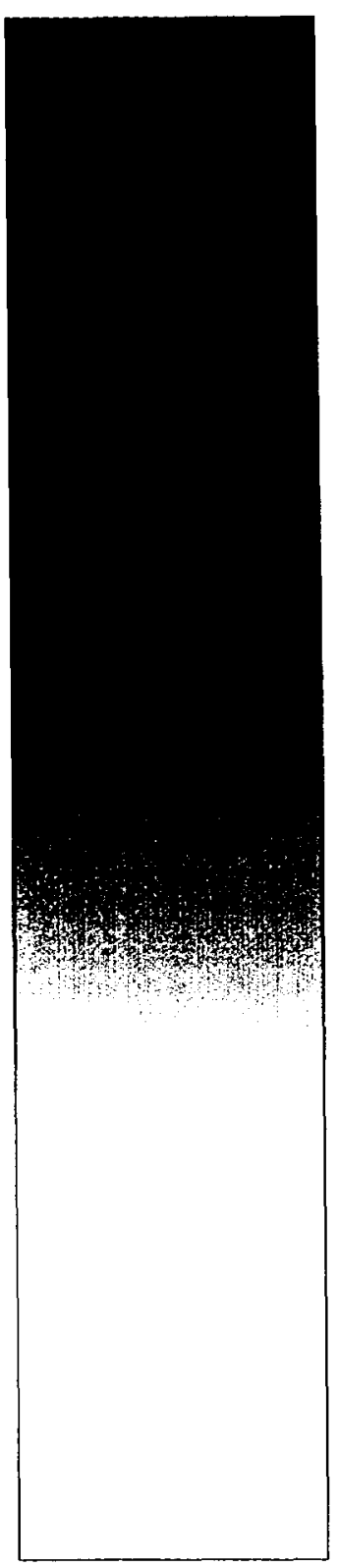

October 2000

Westinghouse Savannah River Company

Distributed to addressee prior to formal review. No further distribution is authorized.

Plutonium Immobilization Project

Lawrence Livermore National Laboratory Livermore, California 94550 
SRT-RSE-2000-00049

October 16,2000

TO: $\quad$ Paul Maddux

703-45A

FROM: $\underset{773-A}{\text { Eric Kriikku }}\{$

\section{CAN LOADING MILESTONE FOR PLUTONIUM IMMOBILIZATION (U)}

Attached are pictures from the Can Loading Puck Can Inspection demonstration. These pictures fulfill the AOP milestone number 2.2.4.5/FY00/b, "Can Loading Robot and Can Swiping Robot

Demonstration Complete." This letter will serve as verification that this milestone is complete.

CC: $\quad$ Bill Randall, 703-45A

Jim Steed, 703-45A

Larry Milton, 703-45A

Clyde Ward, 773-A

Jim Wong, 773-A 


\section{PIP Approval Sheet}

Principal Investigator:

Signature: Linidl 10/24/00 Name:
Approved: SBTC Level 3,Manager

Signature:

Name:

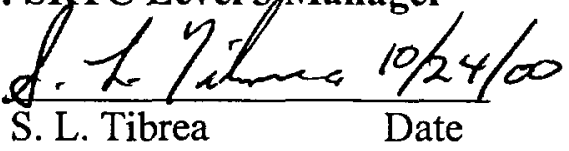

Approved: Task Leader

Signature: Eni trint 10/24/00 Name:
E. M. Kriikku

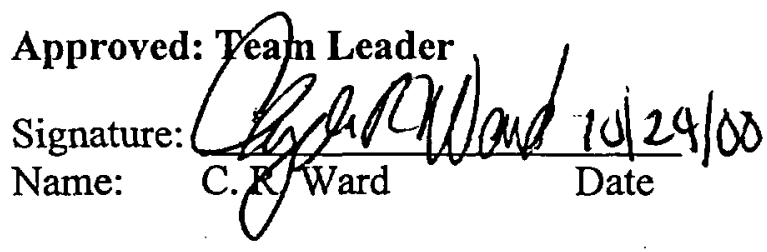

\section{Approved: Activity Leader}

Signature:

Name:
Date

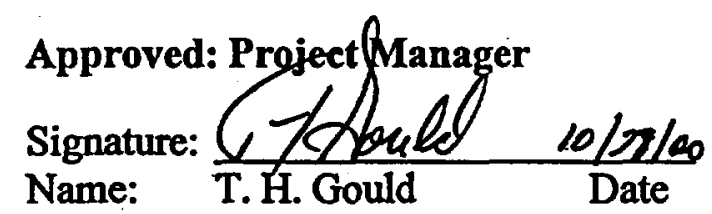

This work was performed under the auspices of the U.S. Department of Energy by the University of California, Lawrence Livermore National Laboratory under Contract No. W-7405-Eng-48. 


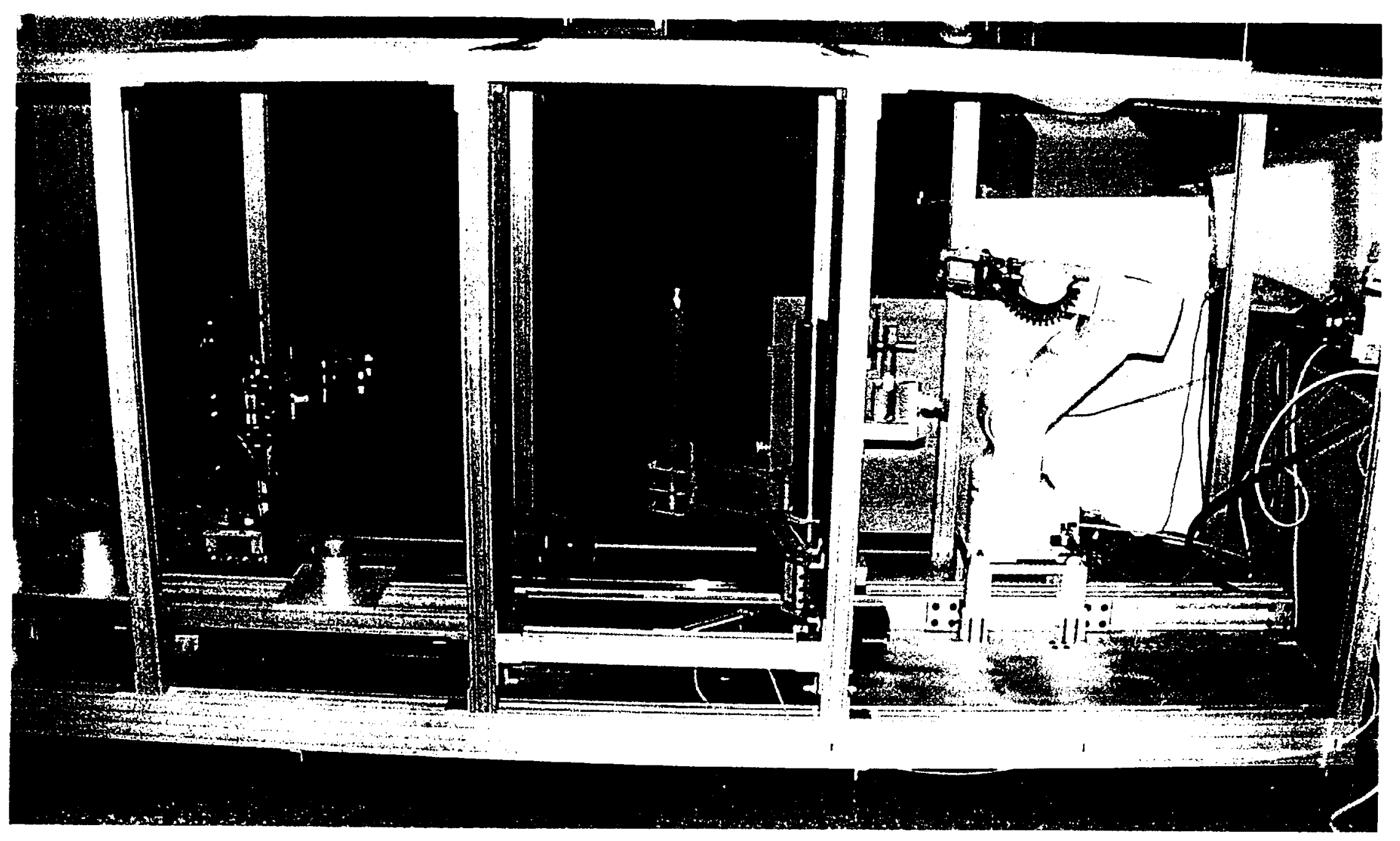

Can Loading - Can Inspection System 


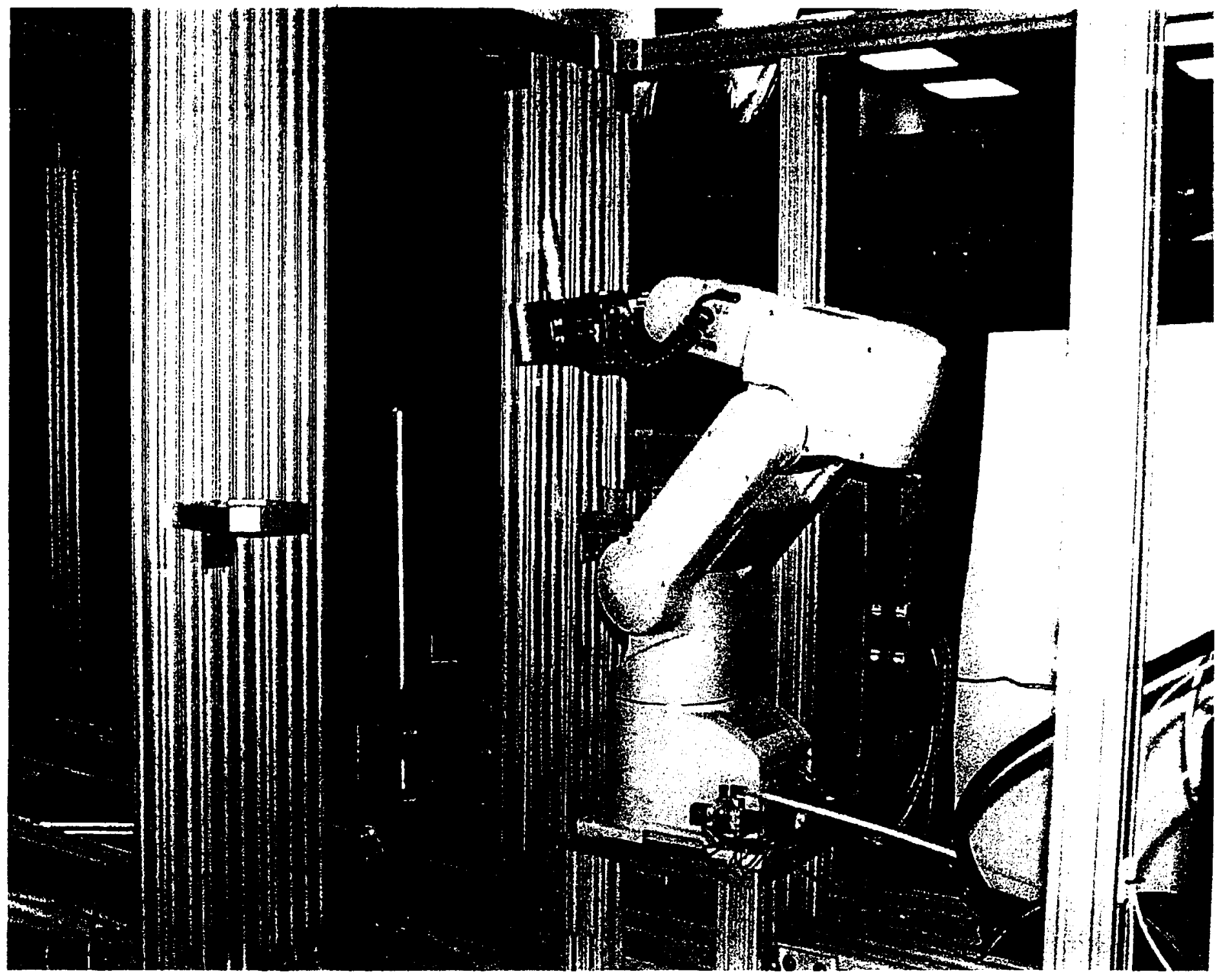

Can Inspection Robot Brings Survey Tool to Puck Can 


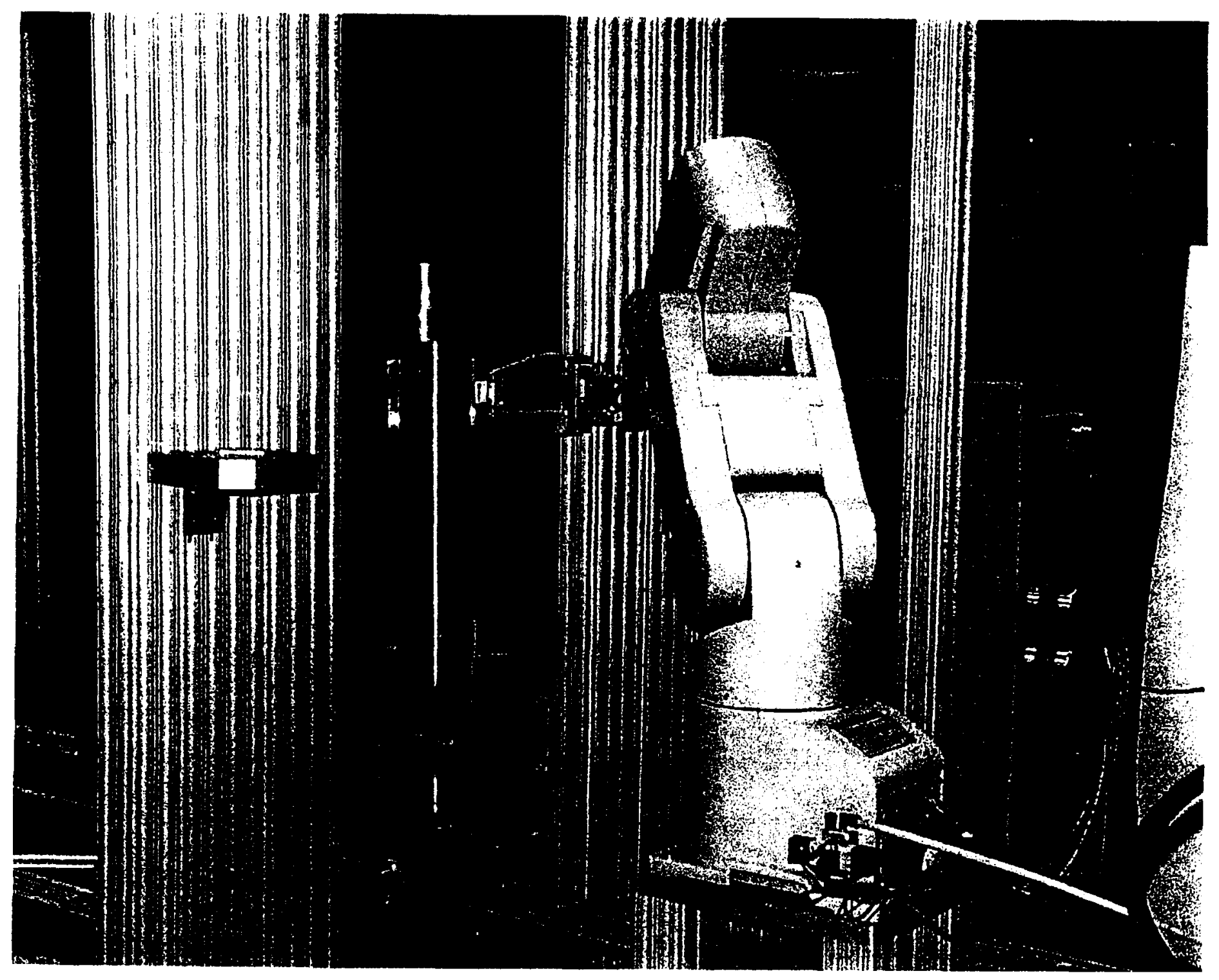

Can Inspection Robot Surveys Puck Can 


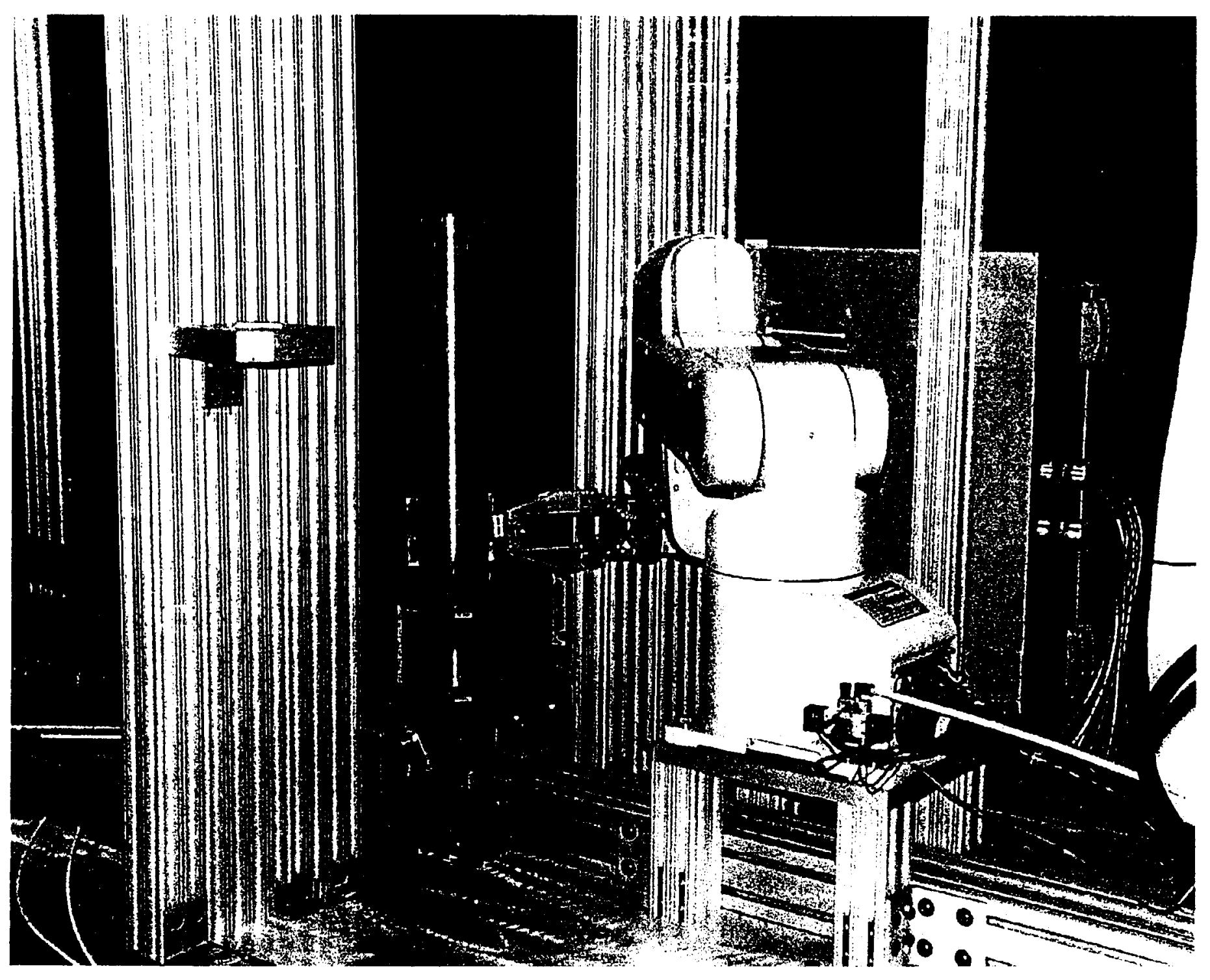

Can Inspection Robot Surveys Puck Can 


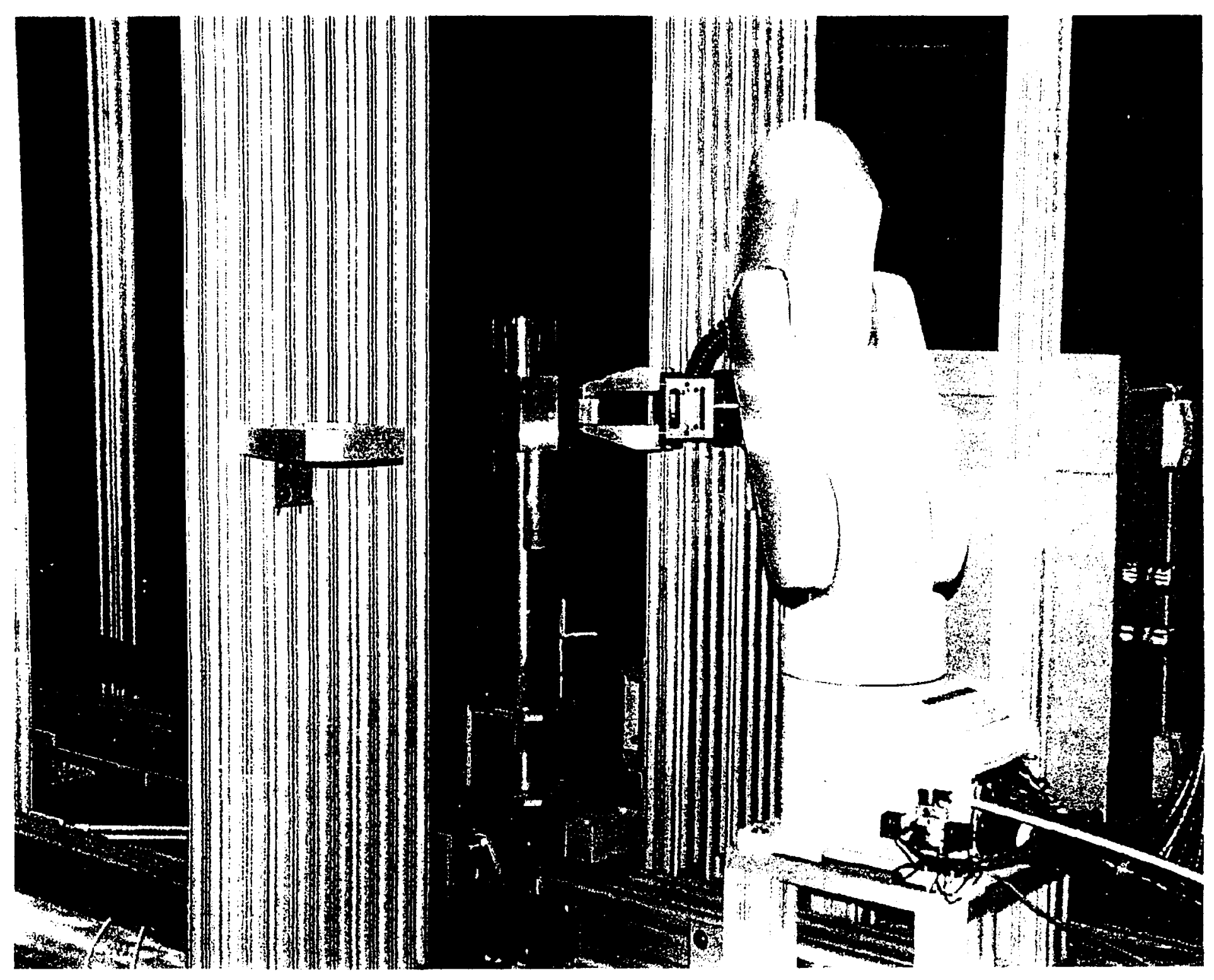

Can Inspection Robot Surveys Puck Can 


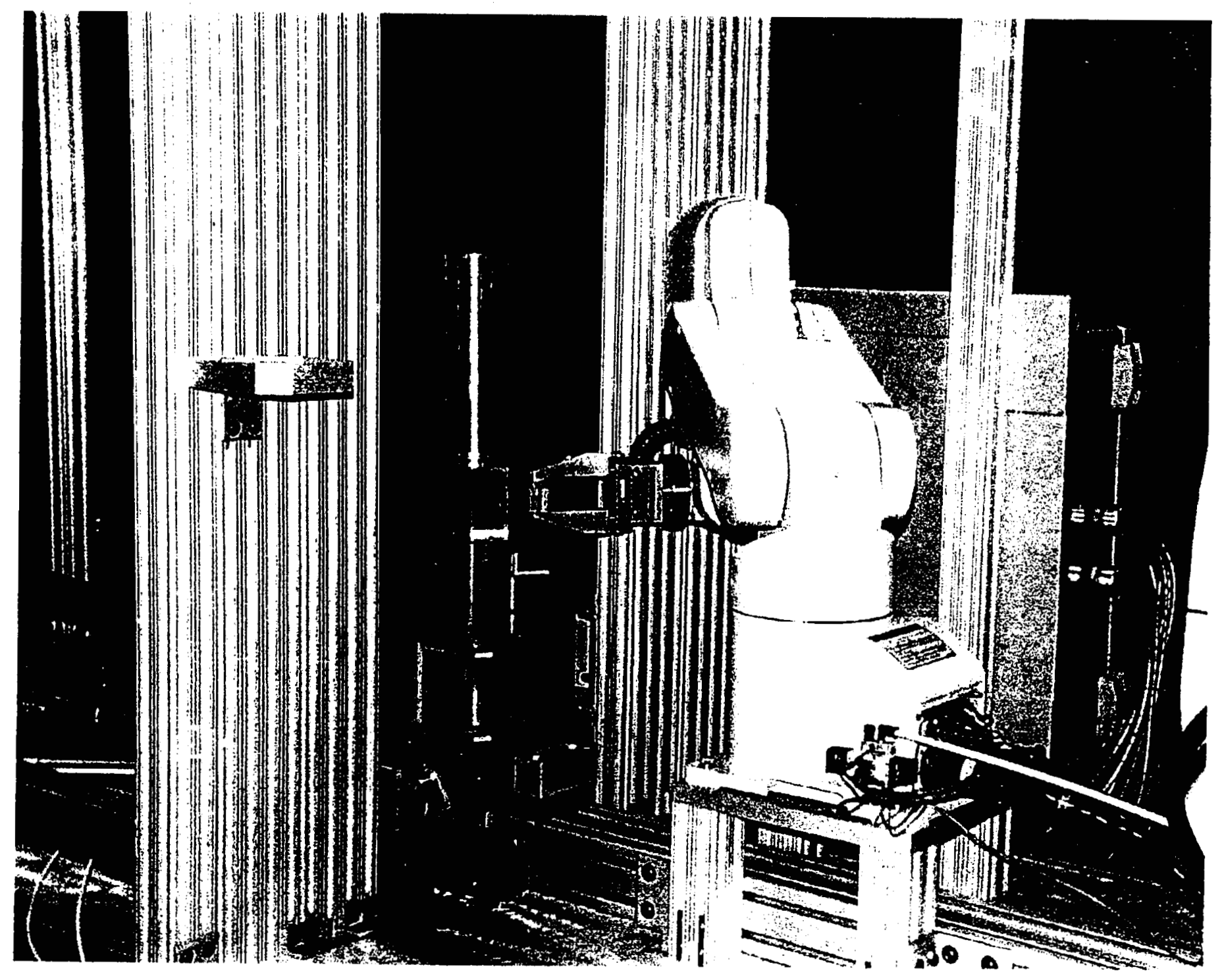

Can Inspection Robot Surveys Puck Can 


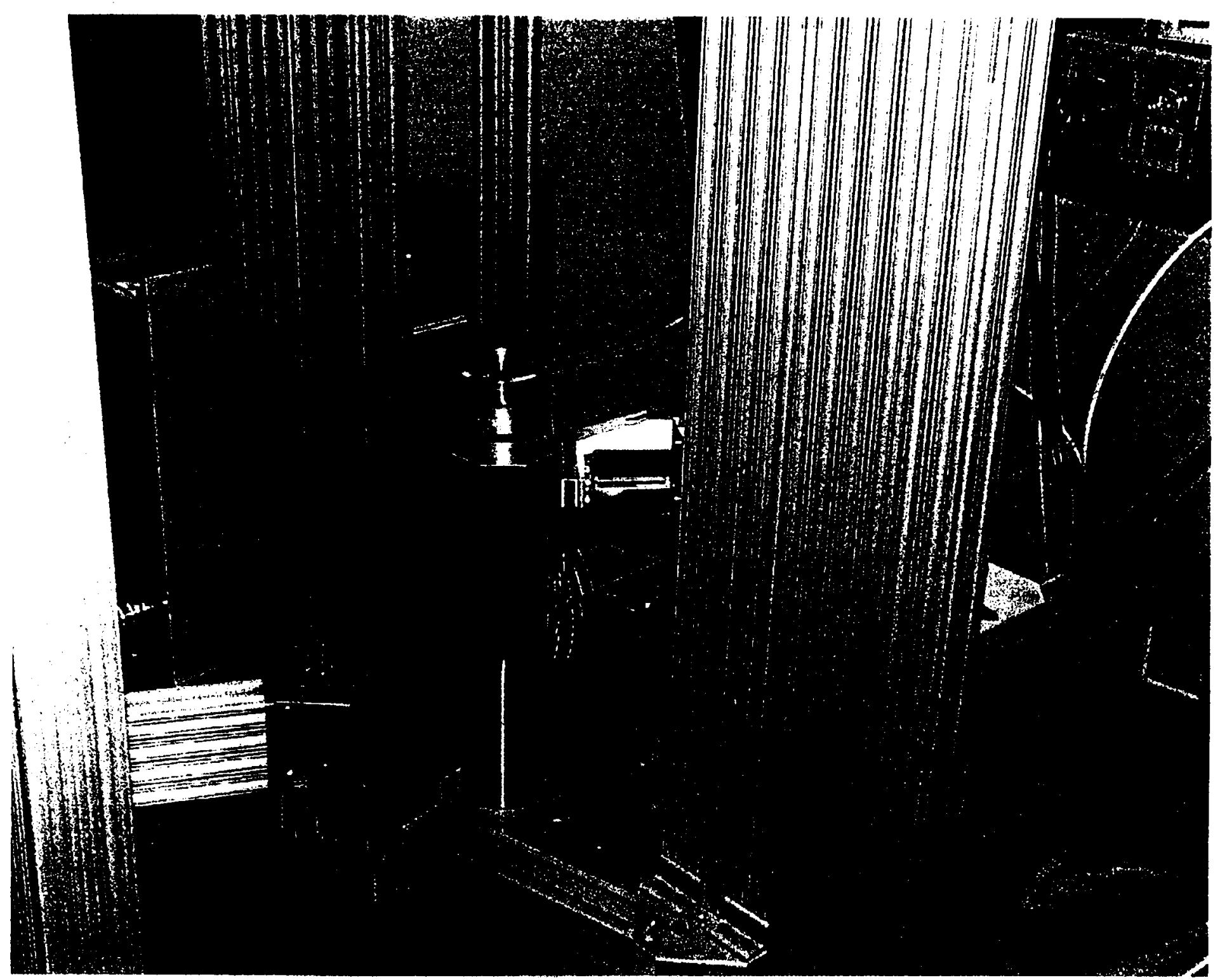

Can Inspection Robot Surveys Puck Can 


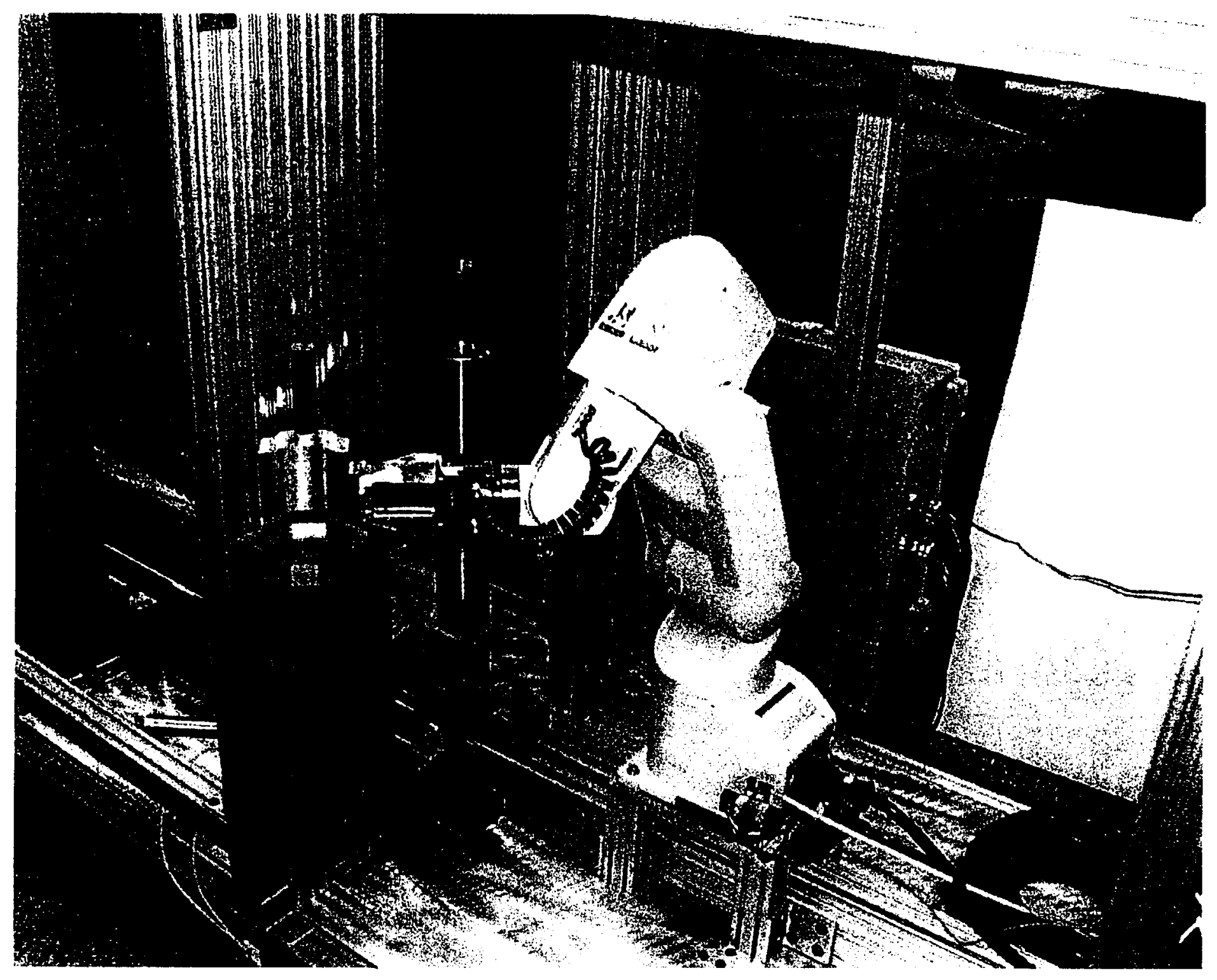

Can Inspection Robot Returns Survey Tool 


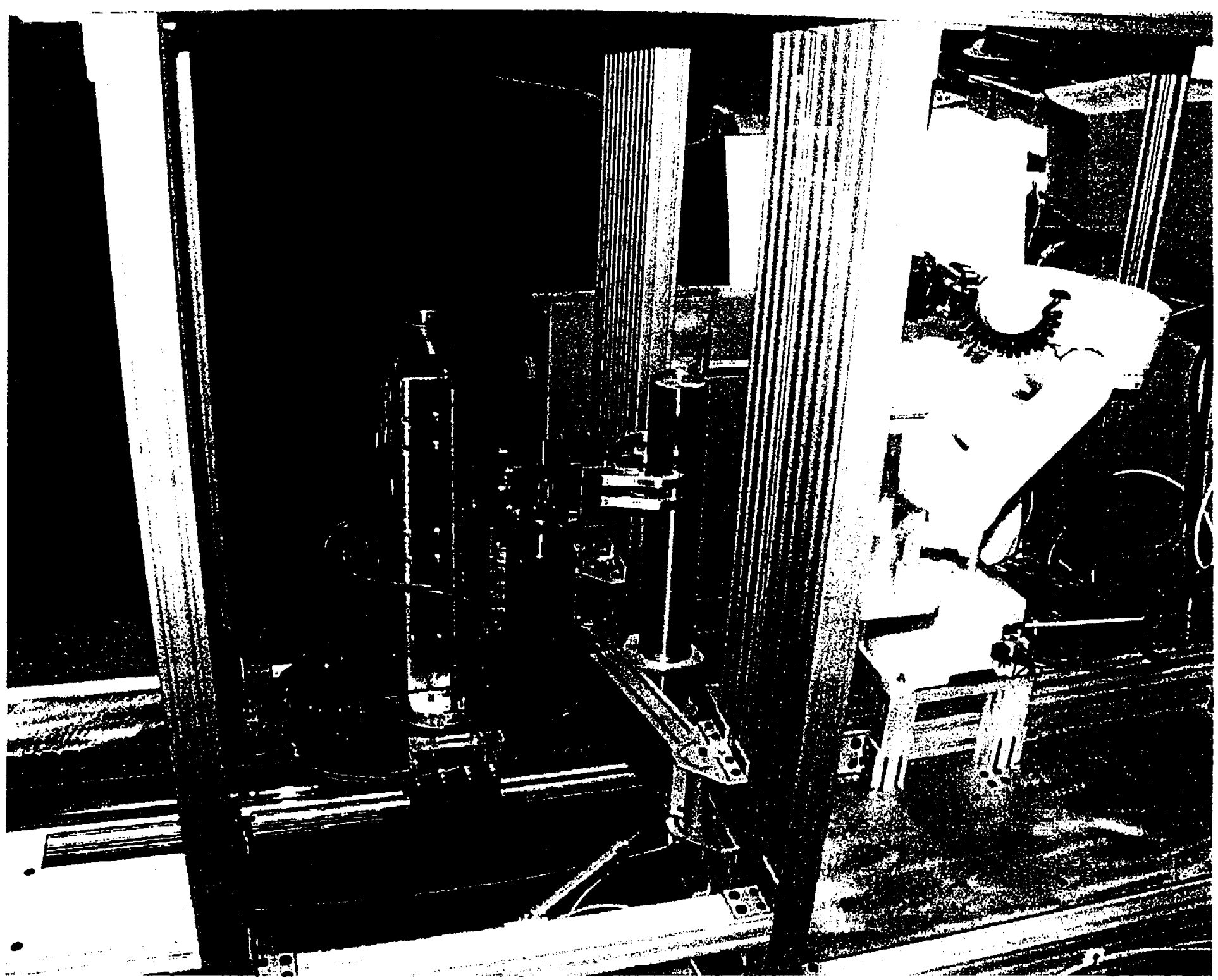

Can Robot Approaches Puck Can 


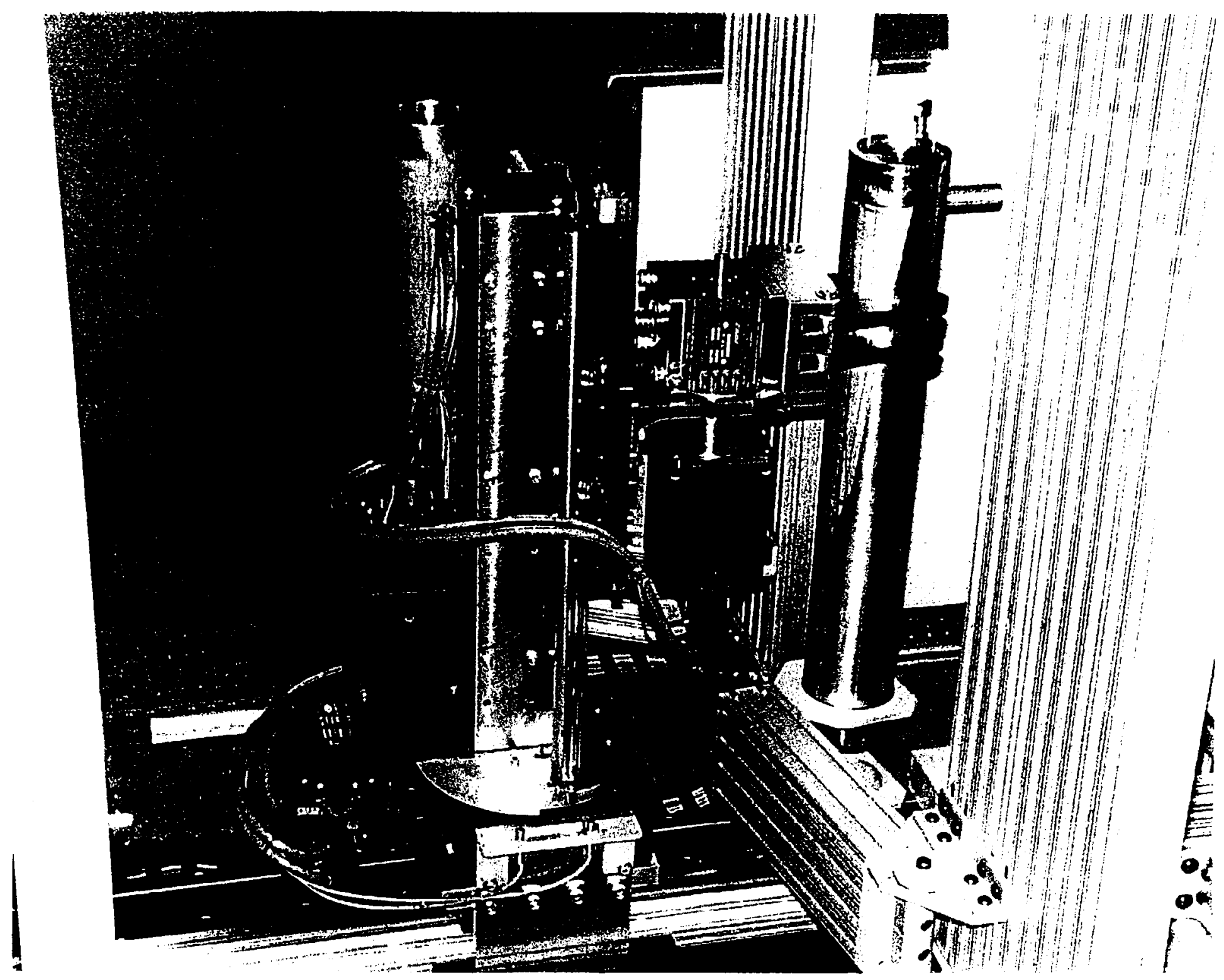

Can Robot Lifts Puck Can from Bagless Transfer Can Holder 


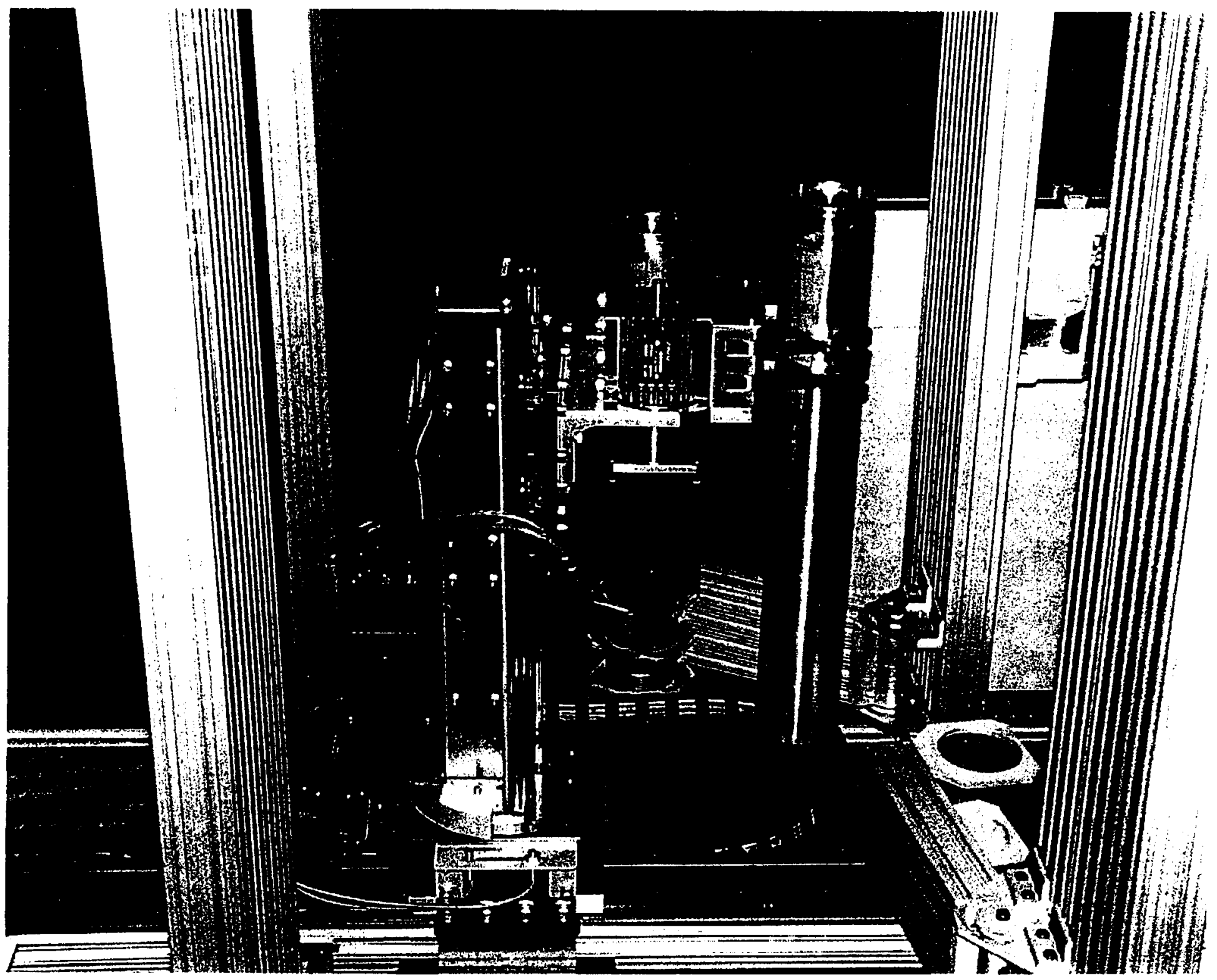

Can Robot Removes Puck Can from Bagless Transfer Can Holder 


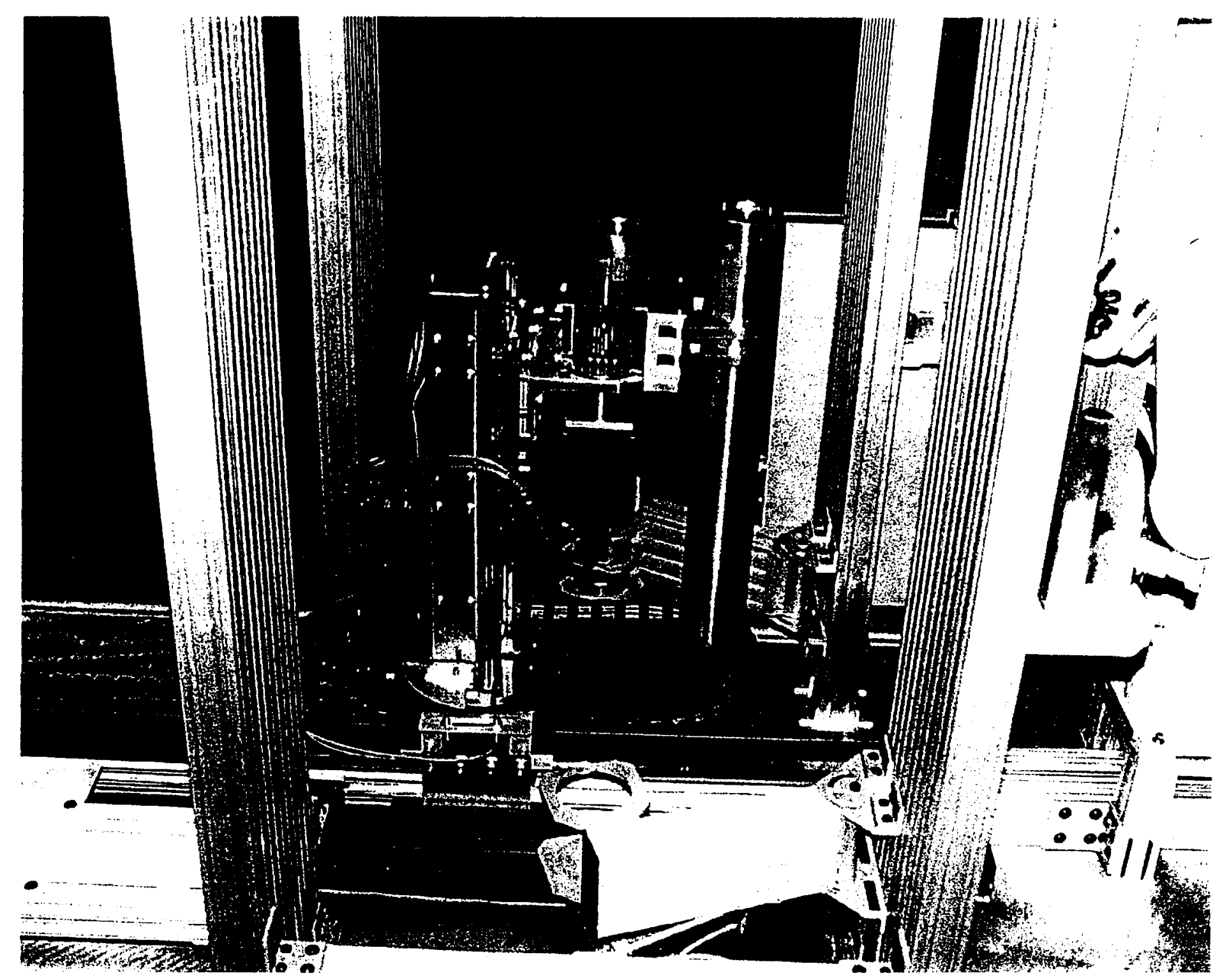

Bagless Transfer Can Holder Retracts 


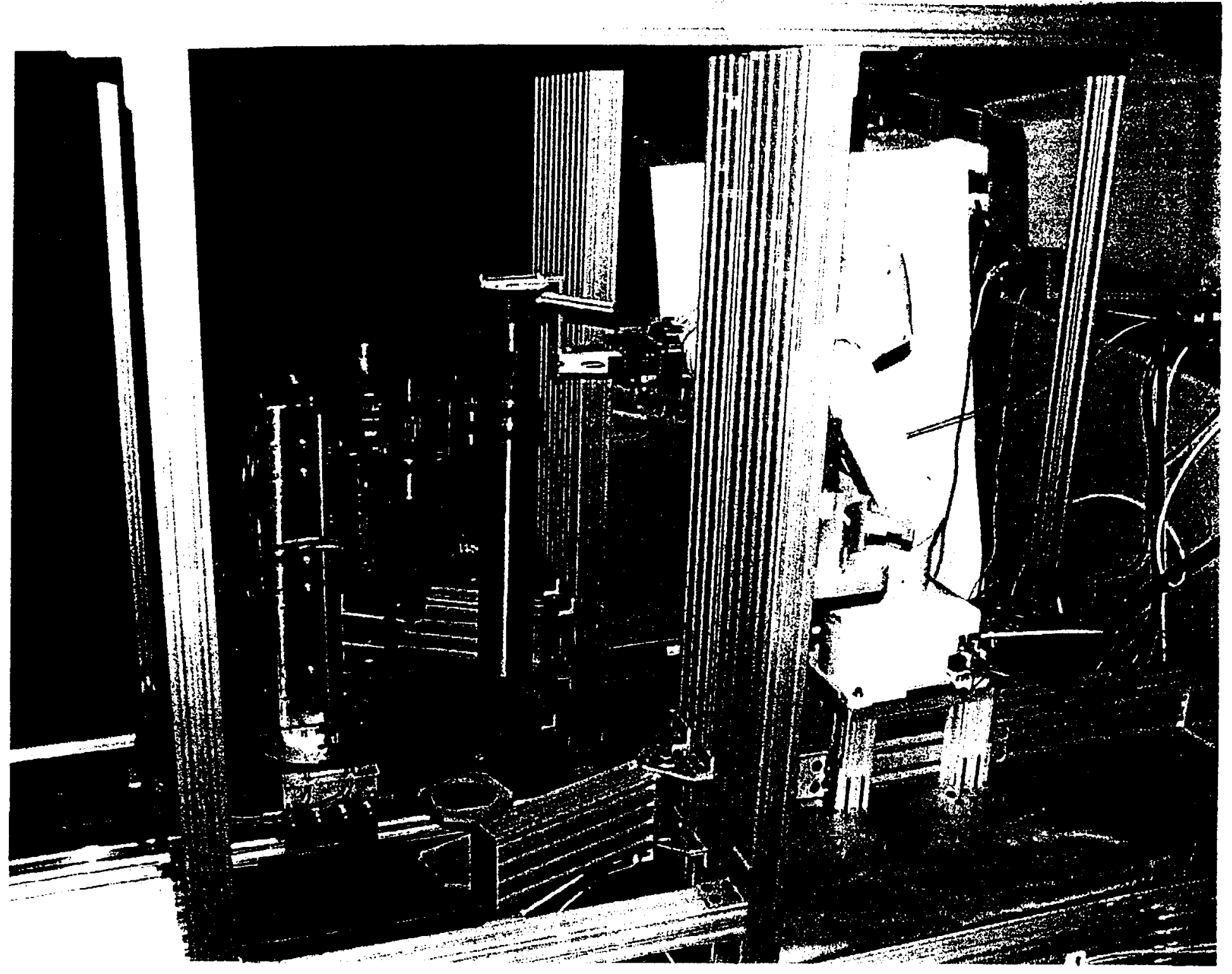

Can Inspection Robot Surveys Puck Can Top 


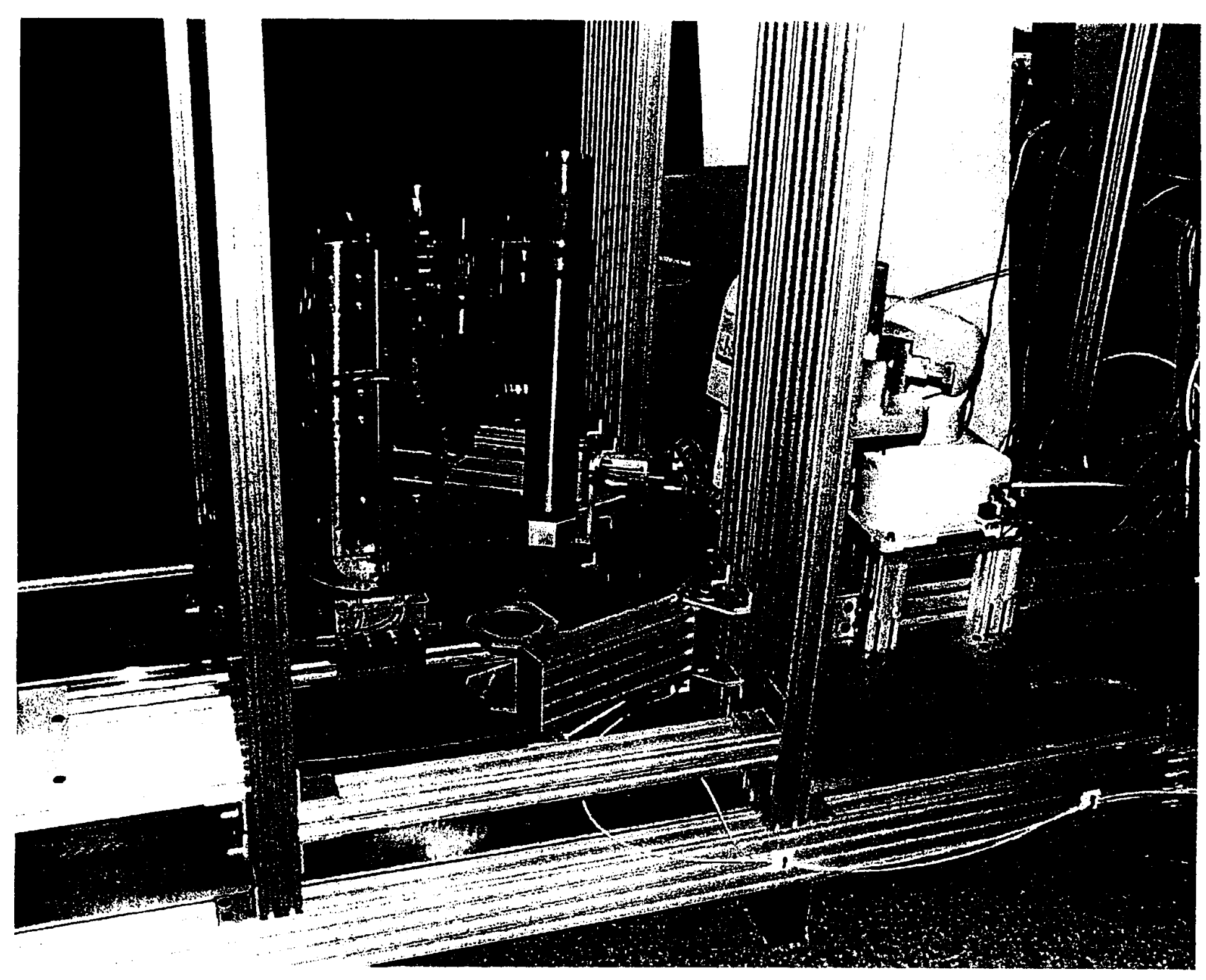

Can Inspection Robot Surveys Puck Can Bottom 


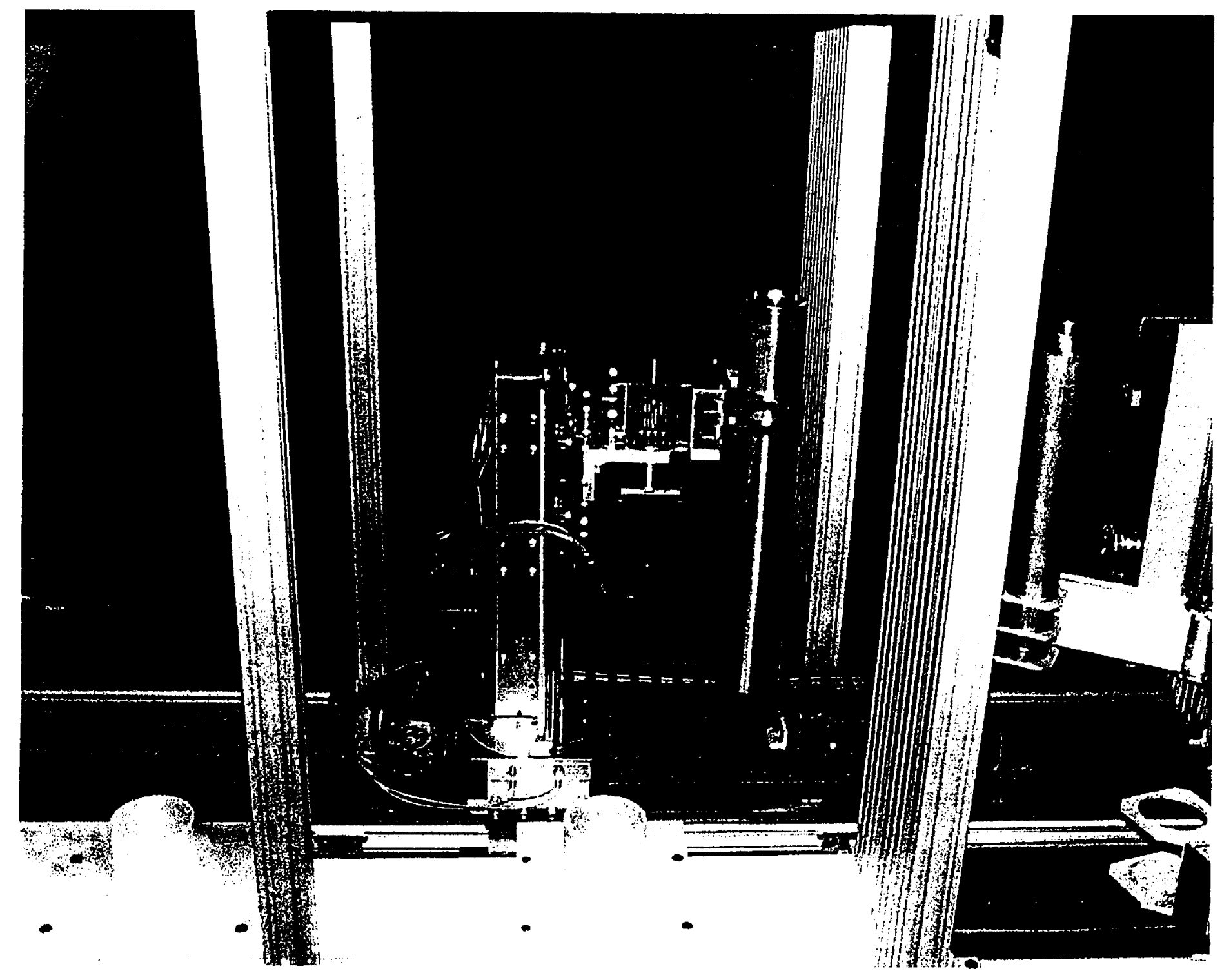

Can Robot Moves Puck Can to Leak Detector 


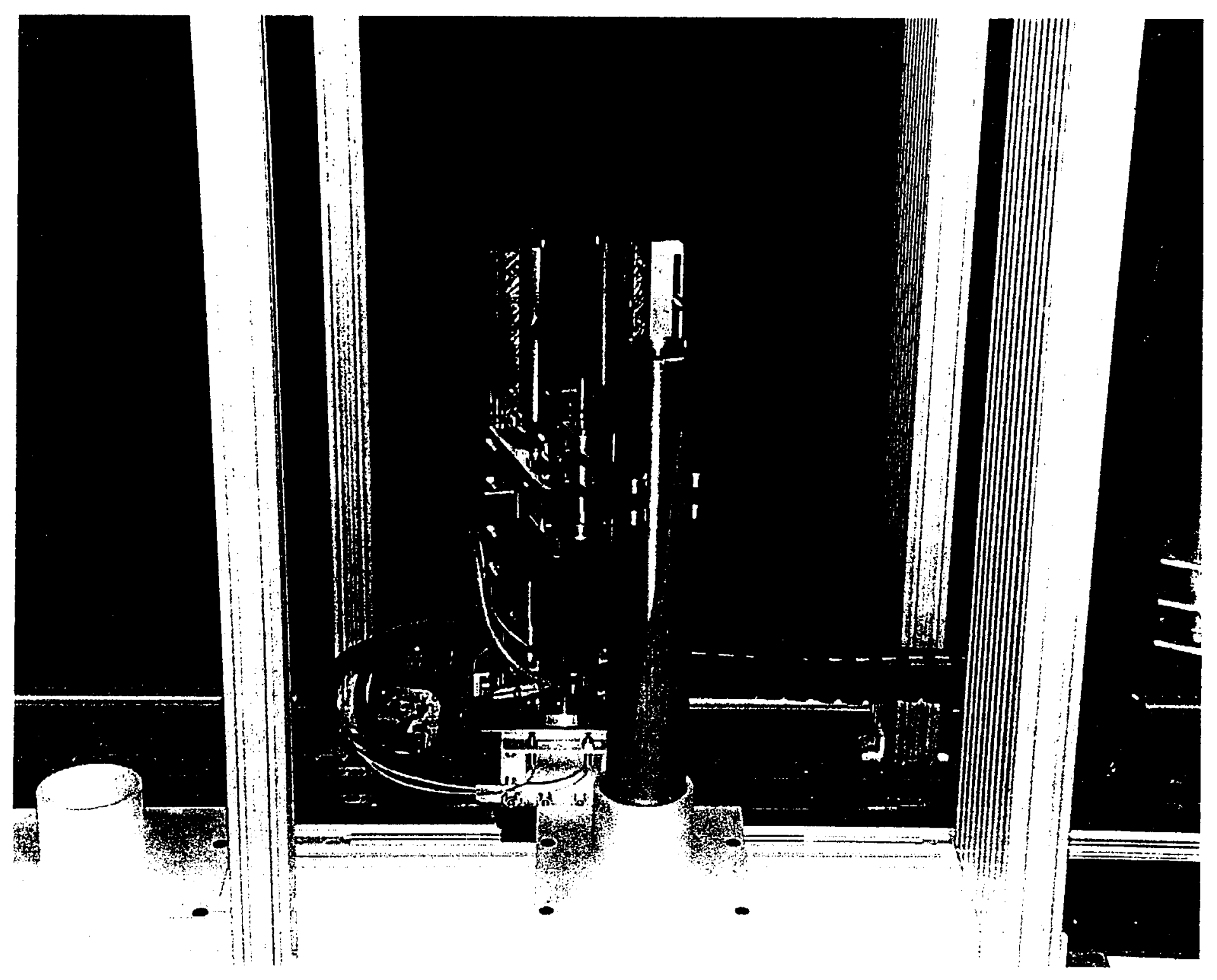

Can Robot Moves Puck Can into Leak Detector 


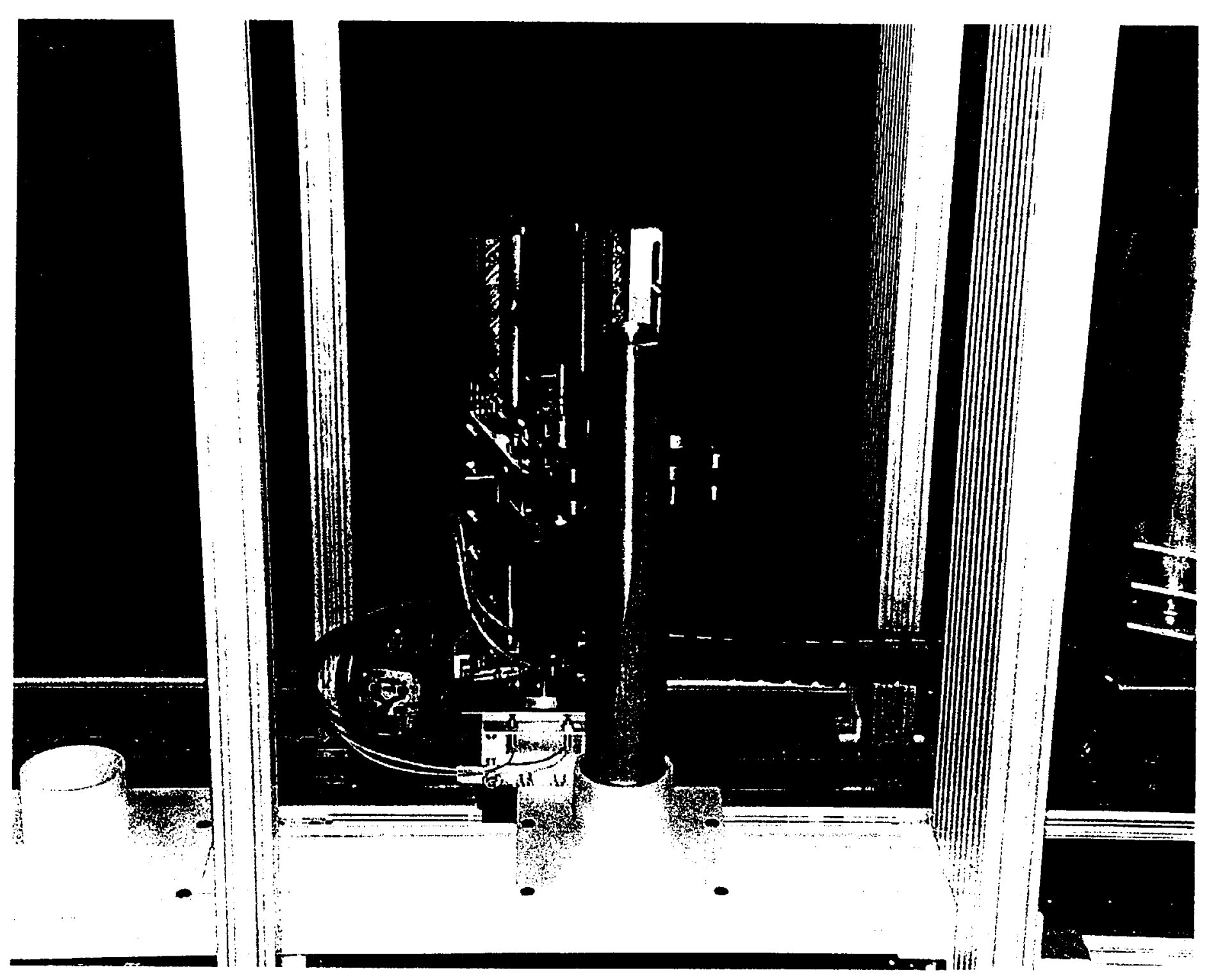

Can Robot Leaves Puck Can in Leak Detector 


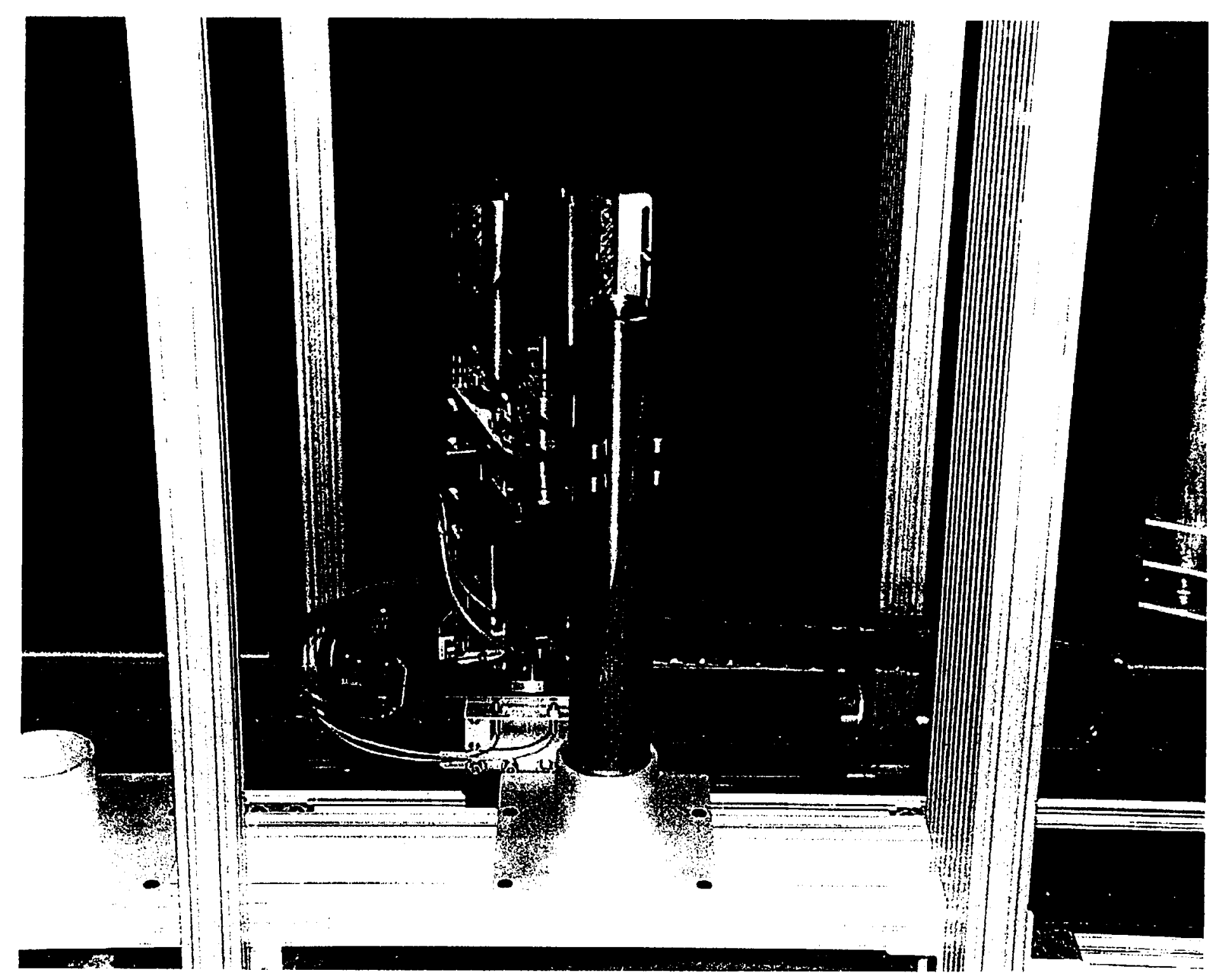

Can Robot Grabs Puck Can 


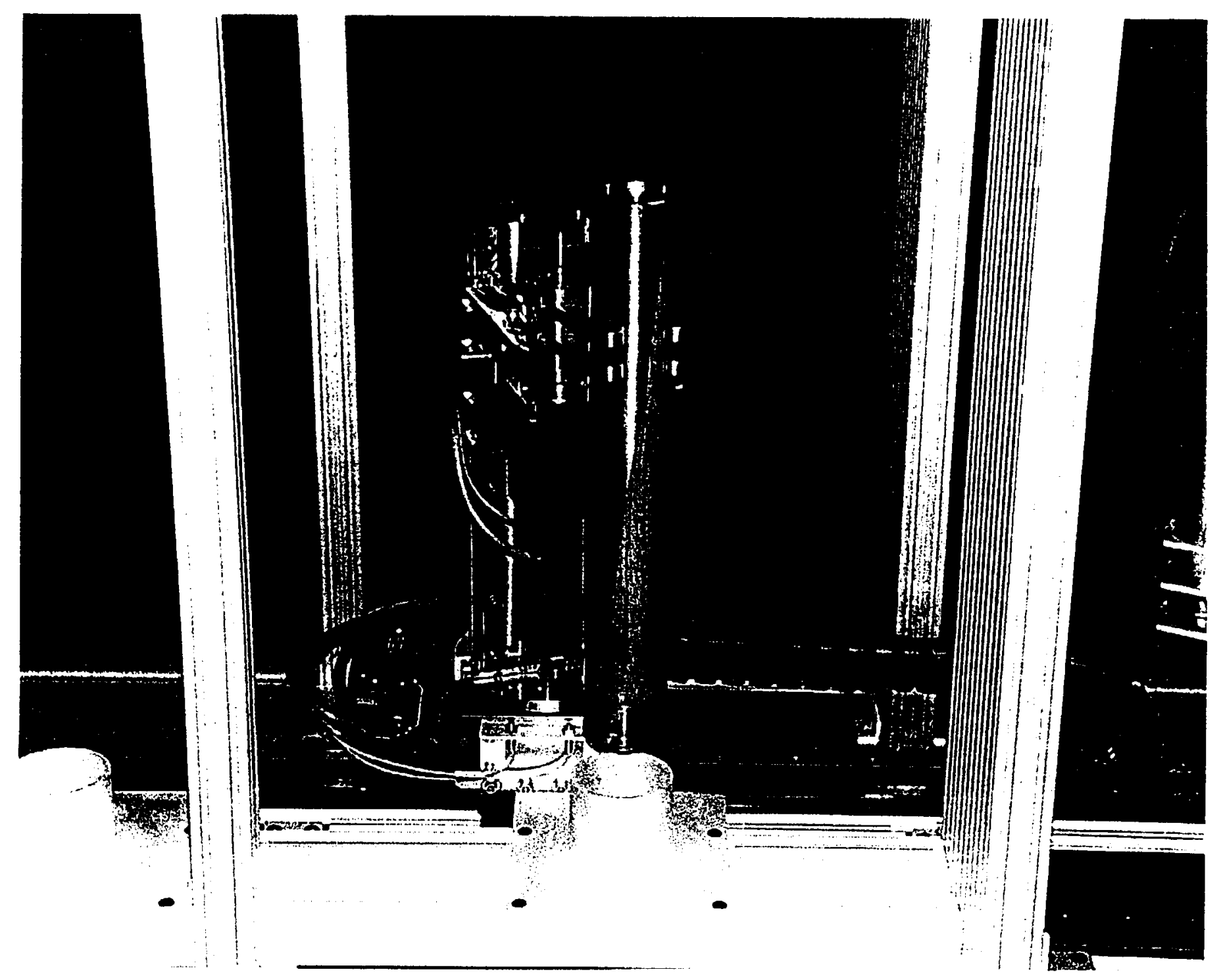

Can Robot Removes Puck Can From Leak Detector 


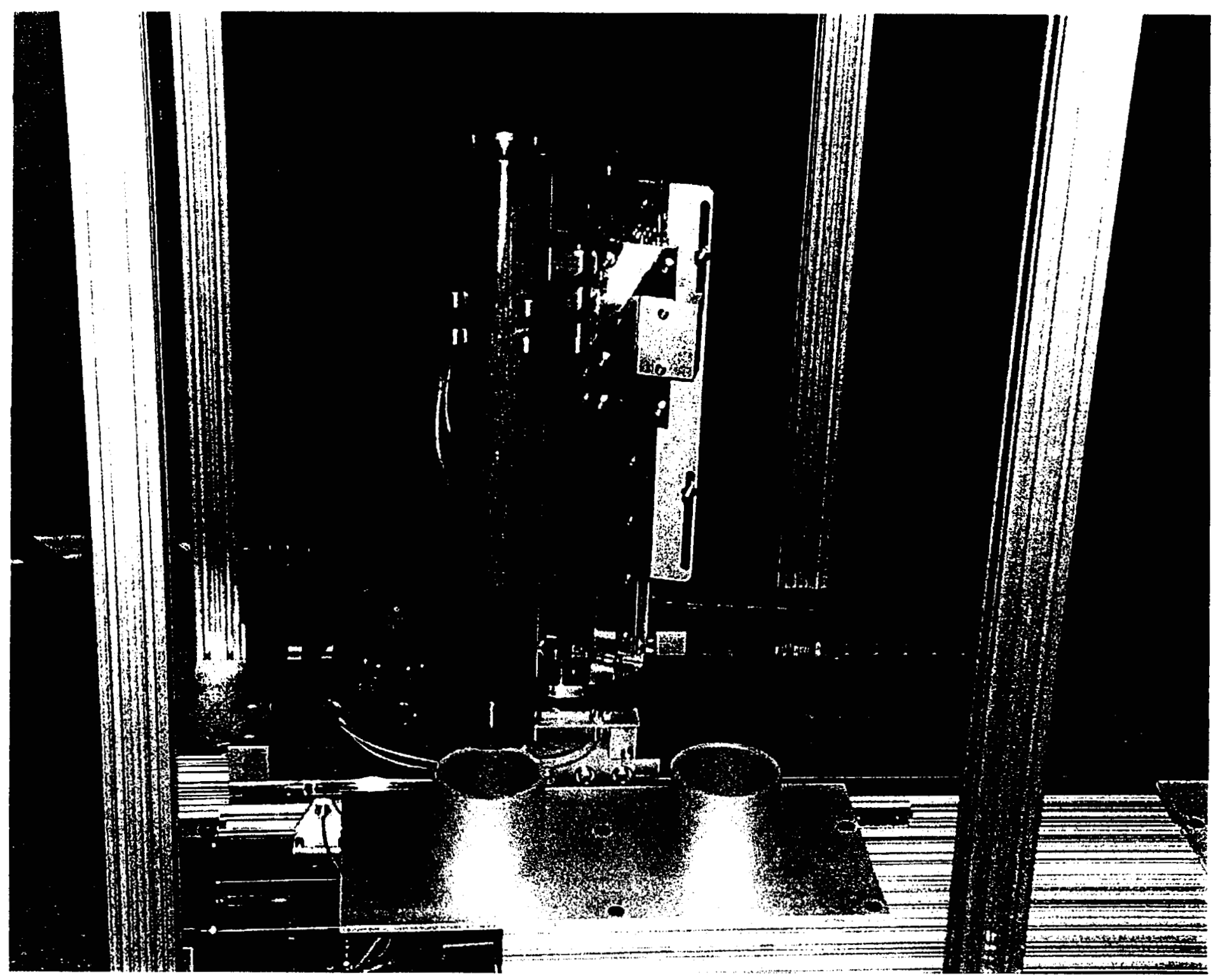

Can Robot Moves Puck Can To Transfer Cart 


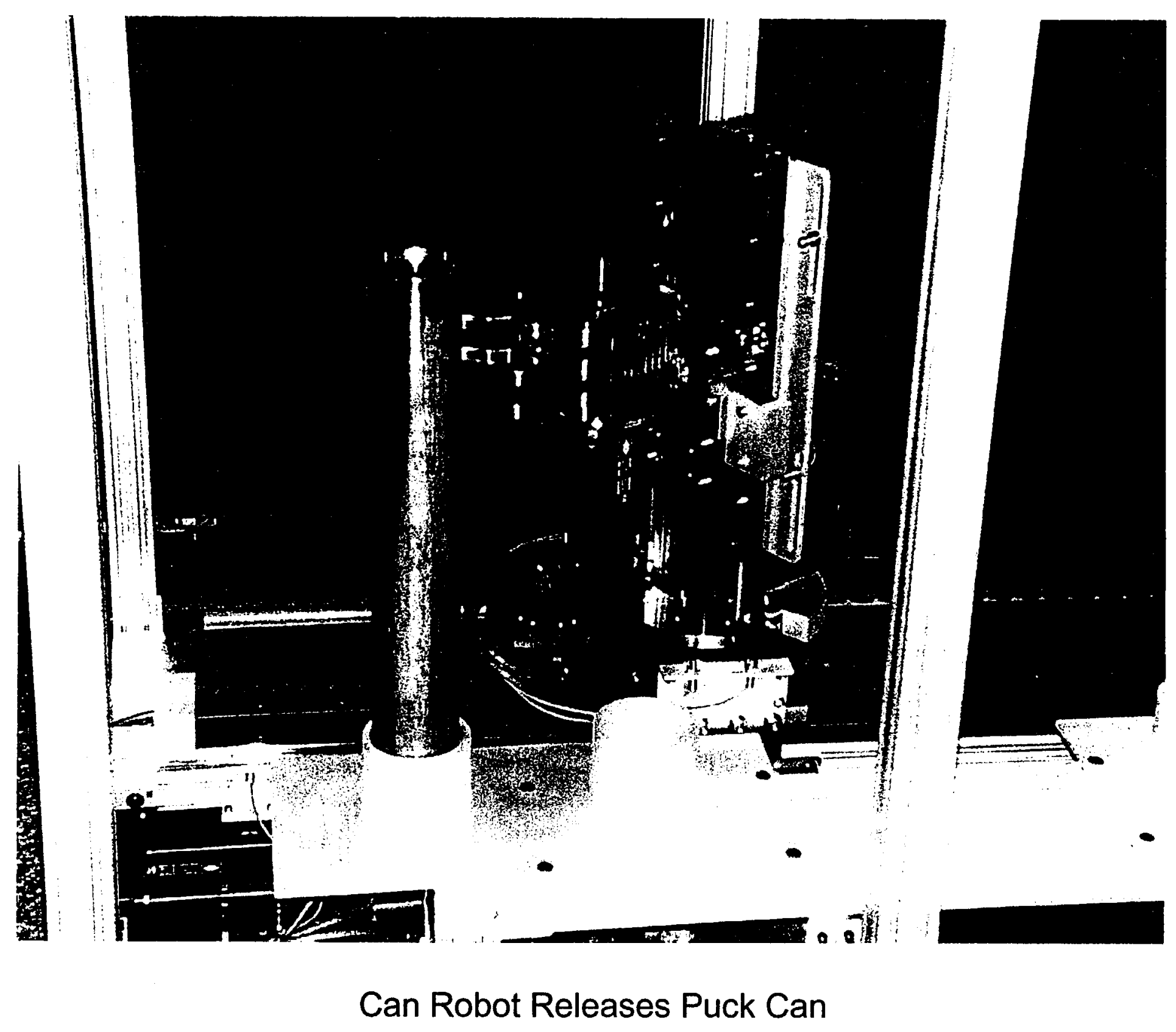




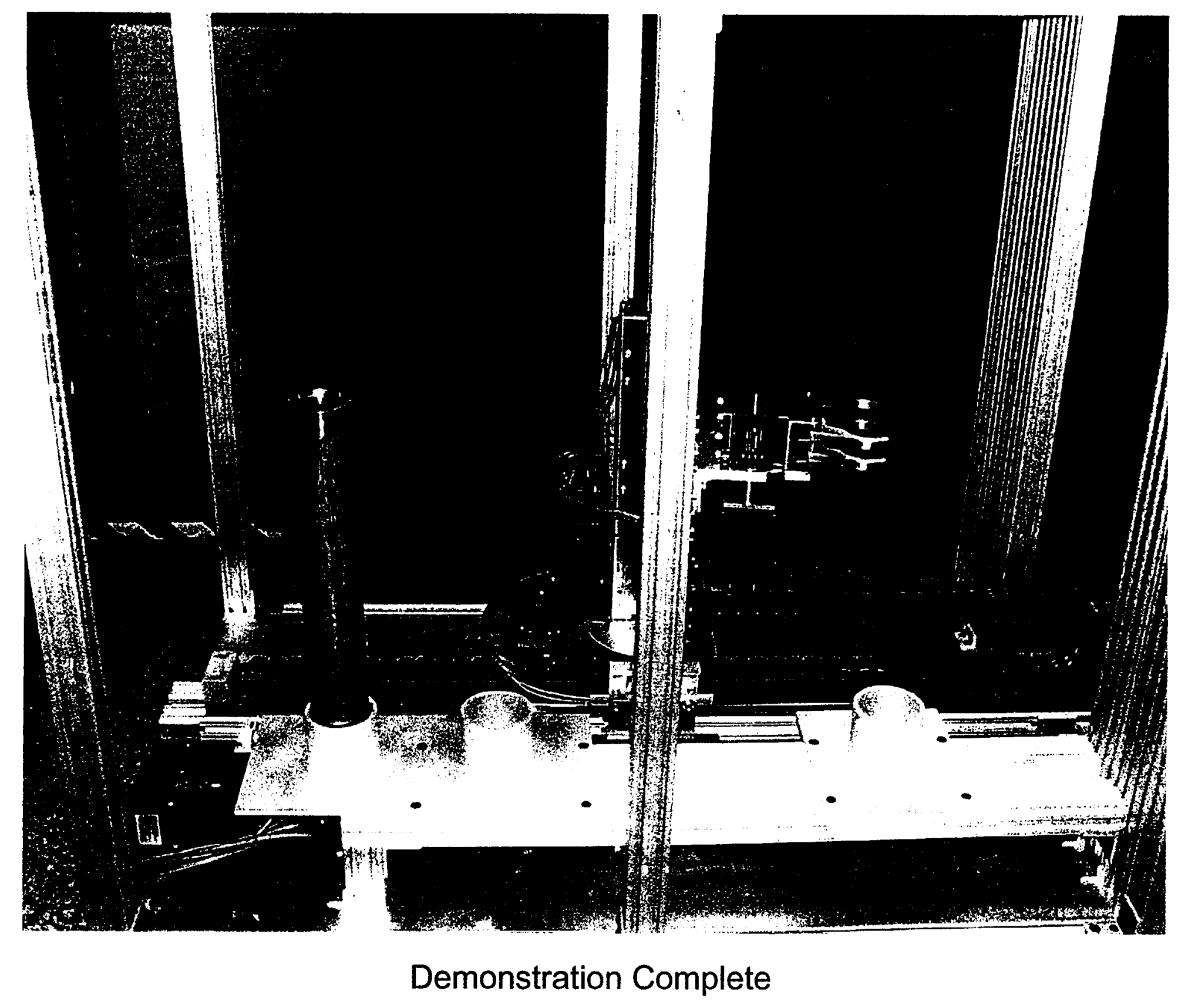

